(1)

CrossMark

\title{
Airway smooth muscle may drive mucus hypersecretion in asthma
}

\author{
James G. Martin ${ }^{1,2}$ \\ Affiliations: 'Dept of Medicine, Division of Respiratory Medicine, Faculty of Medicine, McGill University, \\ Montreal, QC, Canada . ${ }^{2}$ Meakins-Christie Laboratories, Research Institute of the McGill University Health
} Centre, Montreal, QC, Canada.

Correspondence: James G. Martin, Meakins-Christie Laboratories, Research Institute of the McGill University Health Centre, 1001 Decarie Blvd, Montreal, QC, H4A 3J1, Canada. E-mail: james.martinamcgill.ca

@ERSpublications

New insights into a potential property of airway smooth muscle cells with relevance to asthma http://ow.ly/uVe030kPoNN

Cite this article as: Martin JG. Airway smooth muscle may drive mucus hypersecretion in asthma. Eur Respir J 2018; 52: 1801166 [https://doi.org/10.1183/13993003.01166-2018].

The contribution of airway smooth muscle (ASM) to asthma is critical but the multiple ways in which it may contribute to its pathobiology is still an active area of exploration. Contractile, proliferative and secretory properties relevant to asthma have been assessed. To date, studies of the contractile properties of isolated ASM have failed to demonstrate alterations in contractile properties that could explain excessive airway narrowing and airway hyperresponsiveness $[1,2]$, however tempting it is to attribute these features of asthma to intrinsic abnormalities of the muscle. In contrast to studies of tissue strips, cultured ASM cells demonstrate a range of "pro-asthmatic" properties that differ between cells derived from asthmatic subjects and healthy controls. For example, cultured ASM cells show enhanced stiffening when stimulated with contractile agonists [3] and proliferate more rapidly when treated with growth factors [4, 5], a property that would potentially favour a predisposition to remodelling. The pro-inflammatory phenotype is manifest as the expression of cytokines such as the neutrophilic chemoattractant CXCL8, attributable to enhanced NF- $\mathrm{KB}$ binding to the promoter site of CXCL8 [6]. Matrix proteins affecting ASM function and other cellular phenotypes are also secreted by ASM and are determinant of the ASM phenotype (reviewed in [7]).

In the current issue of the European Respiratory Journal, FAIz et al. [8] describe an elegant series of studies using in vitro techniques to study the effects of interleukin (IL)- $1 \beta$ on ASM cells, coupled with the analysis of sputum samples from human asthmatics, which have led to the conclusion that ASM cells contribute to mucus hypersecretion by the epithelium. An examination of gene expression profiles of ASM cells harvested from asthmatic and healthy subjects stimulated with IL-1 $\beta$ revealed that CCL20 and MIR146A were among the genes whose expression was affected by the cytokine. MIR146A was differentially regulated in ASM cells harvested from moderate asthmatics, with a lesser increase in expression and concurrently a greater increase in CCL20 protein secretion by the ASM cells compared to mild asthmatics and healthy controls. The gene product miR-146a-5p was linked to the regulation of the expression of CCL20 by showing that CCL20 expression was reduced by overexpression of miR-146a-5p in the ASM cells. Recombinant CCL20 applied to airway epithelial cells induced mucus production and is a postulated cause of chronic mucus hypersecretion in asthma. The increased level of CCL20 in the sputum of asthmatics provides evidence to support plausibility of the hypothesis. 
MicroRNAs (miRNAs) are small noncoding RNAs that regulate gene expression through binding to the $3^{\prime}$ untranslated region of targeted mRNAs to inhibit protein translation or degradation of mRNAs. miR-146a-5p, which was focused upon by FAIz et al. [8], has been identified in prior publications as an anti-inflammatory miRNA that inhibits the NF- $\kappa B$ downstream signalling molecules IRAK1 and TRAF6 [9] and has been associated with a variety of cancers, including bronchogenic carcinoma. ASM cells harvested from asthmatics stimulated by a combination of cytokines (tumour necrosis factor (TNF)- $\alpha$, IL- $1 \beta$ and interferon- $\gamma$; so-called cytomix) have been previously shown to express higher levels of miR-146-5p than control cells and to negatively regulate IL-1 $\beta$ and cyclooxygenase- 2 synthesis [10]. However, the association of miR-146a-5p with the control of CCL20 synthesis by ASM is a novel finding. CCL20 has been identified also as a product of airway epithelial cells exposed to particulates and its secretion is mitogen-activated protein kinase dependent [11]. It is elevated in the sputum of subjects with chronic obstructive pulmonary disease but in this context has been linked to increase in dendritic cell numbers in the airways [12] and not linked to mucus hypersecretion as suggested in the current article by FAIz et al. [8]. Whether the ASM cells have a greater capacity to secrete CCL20 than epithelial cells and whether the site of action on its receptor CCR6 is at the basolateral rather than apical surface requires further exploration.

Chronic mucus hypersecretion is a feature of some asthmatic phenotypes. The histopathological descriptions of the asthmatic airway highlight mucus cell metaplasia and mucus gland hyperplasia [13]. In vitro studies of airway epithelial cells stimulated with cytokines such as TNF- $\alpha$ have shown an increase in goblet cells in an epidermal growth factor receptor-dependent fashion [14]. Allergen-driven mucus cell hyperplasia has also been shown to be driven by the epidermal growth factor receptor in small animal models of asthma [15]. However, the contribution of mucus hypersecretion to airway obstruction, although suspected, does not appear to have been examined quantitatively until recently. Quantification of mucus plugs in the airways of subjects with asthma by computed tomographic lung scans demonstrated frequent obstructing mucus plugs that tended to persist in the same airways over time and increased in frequency with asthma severity measured by the forced expiratory volume in $1 \mathrm{~s}$ [16].

The place of IL-1 $\beta$ in asthma is being better defined and it is associated with the neutrophilic phenotype of severe asthma [17]. A transcriptomic analysis of sputum from asthmatic subjects has confirmed a phenotype that showed a gene expression signature indicative of involvement of the nucleotide-binding oligomerisation domain, leucine-rich repeat and pyrin domain-containing 3 (NRLP3) inflammasome members and their product, IL-1 $\beta$. It is therefore plausible that IL-1 $\beta$ may drive mucus secretion in this asthmatic phenotype, although the link has not yet been made.

Although the current study by FAIz et al. [8] provides insights into another potential property of ASM cells with relevance to asthma, confirmation in intact humans of the implications of findings from the current study and other reductionist experiments is required. Administration of an appropriate antagonist, even if it reduces sputum volume, will not determine the cells of origin of CCL20. Thus, we are forced to draw conclusions of guilt by association for IL-1 $\beta$-driven relative reductions in miR-146a-5p, CCL20 synthesis and mucus hypersecretion in asthmatic subjects, pending more direct proof. Furthermore, the administration of molecules designed to inhibit mucus secretion would need to be done with caution as the pathophysiological role of mucus is not clear and may indeed have a protective role in some patients. However, for those in whom mucus plugs occur and occlude airways, such a strategy may well be valuable.

Conflict of interest: None declared.

\section{References}

1 Chin LY, Bossé Y, Pascoe C, et al. Mechanical properties of asthmatic airway smooth muscle. Eur Respir J 2012; 40: 45-54.

2 Ijpma G, Kachmar L, Matusovsky OS, et al. Human trachealis and main bronchi smooth muscle are normoresponsive in asthma. Am J Respir Crit Care Med 2015; 191: 884-893.

3 An SS, Kim J, Ahn K, et al. Cell stiffness, contractile stress and the role of extracellular matrix. Biochem Biophys Res Commun 2009; 382: 697-703.

4 Johnson PR, Roth M, Tamm M, et al. Airway smooth muscle cell proliferation is increased in asthma. Am J Respir Crit Care Med 2001; 164: 474-477.

5 Burgess JK, Ketheson A, Faiz A, et al. Phenotype and functional features of human telomerase reverse transcriptase immortalized human airway smooth muscle cells from asthmatic and non-asthmatic donors. Sci Rep 2018; 8: 805.

6 John AE, Zhu YM, Brightling CE, et al. Human airway smooth muscle cells from asthmatic individuals have CXCL8 hypersecretion due to increased NF- $\mathrm{KB}$ p65, C/EBP $\beta$, and RNA polymerase II binding to the CXCL8 promoter. J Immunol 2009; 183: 4682-4692.

7 Wright DB, Trian T, Siddiqui S, et al. Functional phenotype of airway myocytes from asthmatic airways. Pulm Pharmacol Ther 2013; 26: 95-104.

8 Faiz A, Weckmann M, Tasena H, et al. Profiling of healthy and asthmatic airway smooth muscle cells following interleukin-1 $\beta$ treatment: a novel role for CCL20 in chronic mucus hypersecretion. Eur Respir J 2018; 52: 1800310. 
9 Quinn SR, O'Neill LA. A trio of microRNAs that control Toll-like receptor signalling. Int Immunol 2011; 23: 421-425.

10 Comer BS, Camoretti-Mercado B, Kogut PC, et al. MicroRNA-146a and microRNA-146b expression and anti-inflammatory function in human airway smooth muscle. Am J Physiol Lung Cell Mol Physiol 2014; 307: L727-L734.

11 Reibman J, Hsu Y, Chen LC, et al. Airway epithelial cells release MIP-3 $\alpha /$ CCL20 in response to cytokines and ambient particulate matter. Am J Respir Cell Mol Biol 2003; 28: 648-654.

12 Demedts IK, Bracke KR, Van Pottelberge G, et al. Accumulation of dendritic cells and increased CCL20 levels in the airways of patients with chronic obstructive pulmonary disease. Am J Respir Crit Care Med 2007; 175: 998-1005.

13 Jeffery PK. Remodeling in asthma and chronic obstructive lung disease. Am J Respir Crit Care Med 2001; 164: S28-S38.

14 Takeyama K, Dabbagh K, Lee HM, et al. Epidermal growth factor system regulates mucin production in airways. Proc Natl Acad Sci USA 1999; 96: 3081-3086.

15 Busse PJ, Zhang TF, Schofield B, et al. Decrease in airway mucous gene expression caused by treatment with anti-tumor necrosis factor $\alpha$ in a murine model of allergic asthma. Ann Allergy Asthma Immunol 2009; 103: 295-303.

16 Dunican EM, Elicker BM, Gierada DS, et al. Mucus plugs in patients with asthma linked to eosinophilia and airflow obstruction. J Clin Invest 2018; 128: 997-1009.

17 Rossios C, Pavlidis S, Hoda U, et al. Sputum transcriptomics reveal upregulation of IL-1 receptor family members in patients with severe asthma. J Allergy Clin Immunol 2018; 141: 560-570. 